\begin{tabular}{|c|l|}
\hline Title & Why and how do systems react in thermally fluctuating environments? \\
\hline Author(s) & Kawai, Shinnosuke; Komatsuzaki, Tamiki \\
\hline Citation & $\begin{array}{l}\text { Physical Chemistry Chemical Physics, 13(48), 21217-21229 } \\
\text { https://doi.org/40.1039/c1cp22504a }\end{array}$ \\
\hline Issue Date & 2011 -12-28 \\
\hline Doc URL & http://hdl.handle.net/2115/50277 \\
\hline Rights & Phys. Chem. Chem. Phys., 2011, 13, 21217-21229- Reproduced by permission of the PCCP Owner Societies \\
\hline Type & article (author version) \\
\hline File Information & PCCP13_48_21217-21229.pdf \\
\hline
\end{tabular}

Instructions for use 


\title{
Why and how do systems react in thermally fluctuating environments?
}

\author{
Shinnosuke Kawai ${ }^{1}$ and Tamiki Komatsuzaki ${ }^{1}$ \\ ${ }^{1}$ Molecule $\mathcal{E}$ Life Nonlinear Sciences Laboratory, Research Institute for Electronic Science, \\ Hokkaido University, Kita 20 Nishi 10, Kita-ku, Sapporo 001-0020, Japan
}

\begin{abstract}
Complicated trajectories of chemical reaction in multi-dimensional molecular systems with thermally fluctuating environments are disentangled into simple forms.
\end{abstract}

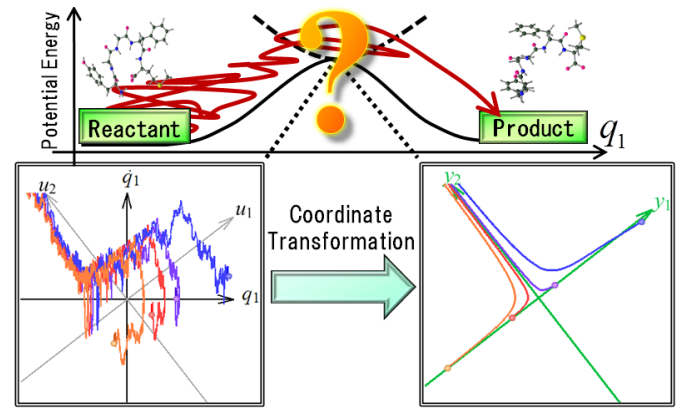

Many chemical reactions, including those of biological importance, take place in thermally fluctuating environments. Compared to isolated systems, there arise markedly different features due to the effects of energy dissipation through friction and stochastic driving by random forces reflecting the fluctuation of the environment. Investigation of how robustly the system reacts under the influence of thermal fluctuation, and elucidating the role of thermal fluctuation in the reaction are significant subjects in the study of chemical reactions. In this article, we start with overviewing the generalized Langevin equation (GLE), which has long been used and continues to be a powerful tool to describe a system surrounded by a thermal environment. It has been also generalized further to treat a nonstationary environment, in which the conventional fluctuation-dissipation theorem no longer holds. Then, within the framework of the Langevin equation we present a method recently developed to extract a new reaction coordinate that is decoupled from all the other coordinates in the region of a rank-one saddle linking the reactant and the product. The reaction coordinate is buried in nonlinear couplings among the original coordinates and the influence of stochastic random force. It was ensured that the sign of this new reaction coordinate (=a nonlinear functional of the original coordinates, velocities, friction, and random force) at any instant is sufficient to determine in which region, the reactant or the product, the system finally arrives. We also discuss how one can extend the method to extract such a coordinate from the GLE framework in stationary and nonstationary environments, where memory effects exist in dynamics of the reaction.

\section{INTRODUCTION}

Understanding of reactions, or more generally the change of states in a multi-body system, is of pivotal importance in a wide range of scientific studies including not just reactions in chemistry, ${ }^{1-5}$ but also ionization of a hydrogen atom in external fields, ${ }^{6}$ isomerization of clusters, ${ }^{7}$ the escape of asteroids, ${ }^{8}$ the diffusion of impurities in crystalline materials, ${ }^{9}$ and the folding/unfolding of proteins. ${ }^{10}$ A reaction can be regarded as a motion of atoms, or more generally of a motion of a point in an appropriate phase space, starting in a certain (phase space) region corresponding to the 'reactants', and going into another region called the 'products'. A main goal in the study of reaction dynamics is to predict, given the present state (the values of position coordinates and momenta) of the system, whether the system will evolve into the products region or the reactants region. In many cases, each of the two regions corresponds to a valley of the potential energy surface of the system. Between the two valleys, there is often a rank-one saddle point, where the potential is maximum along the direction connecting the reactants and the products regions but is minimum along all the other directions. (Note, however, that some reactions, especially highly exothermic reactions, do not possess a saddle point ${ }^{11}$.) The vicinity of the saddle point is sometimes called "the region of the saddle" or "the saddle region." When there is a rank-one saddle point and the energy is not very high, all reactive trajectories necessarily pass from the reactant (product) to the product (reactant) regions through the region of the saddle. Therefore, the dynamics in the saddle region is very crucial for unveiling the cause of reactions, namely, what determines the fate of reactions.

The dynamics in the saddle region has been extensively studied. ${ }^{6,7,12-51}$ A brief (and probably not sufficient) summary may be given by pointing out the importance of two geometrical concepts in the phase space. One is called "Transition State (TS)", defined originally by Wigner as a hyper- 
surface in the phase space between the reactants and the products regions, such that once the system crosses that surface it will fall into the products region without recrossing that surface. The TS has long been postulated to exist ${ }^{12-19}$ but it was just recently that the TS was proved to exist robustly with a firm mathematical ground ${ }^{6,7,20-39}$ following several developments in experiments ${ }^{40,41}$ and theories. ${ }^{42-51}$ Another important object is an invariant manifold, of which there are several kinds. ${ }^{37-39}$ An invariant manifold is a set of points in the phase space such that any point in that set will perpetually remain in that set during the time evolution. As a consequence, if an invariant manifold divides the whole phase space into two disjoint regions, the system can never cross the invariant manifold from one region to the other. An important invariant manifold in the context of reaction dynamics is the one that separates the trajectories going into the product region from those going into the reactant region. Once we know which side of the manifold a given initial condition is, we can tell without any further time propagation whether the reaction occurs or not. In what follows, this manifold between the reactive and the non-reactive trajectories will be called a "reactivity boundary", distinguishing it from a "reaction boundary" which may signify the TS, the no-recrossing surface between the reactants and the products. Recently it was shown that the invariant manifold dividing the trajectories into reactive and nonreactive ones can be extracted analytically even when the nonlinearity of the system is so large that the concept of noreturn TS is not applicable. ${ }^{52,53}$

Many important reactions in biology and industry occur in condensed phase. A characteristic feature of condensed phase reactions is that they involve a huge number of atoms, including the atoms in the solvent molecules. Although numerical simulation on the all-atom basis is becoming possible through the development of computational technology, it is desirable to find a low-dimensional description for the purpose of understanding. A representation called the generalized Langevin equation (GLE) has thus been developed, ${ }^{54-56}$ in which the total system is decomposed into a system with a small number of selected variables and a bath (or environment) that represents the collection of all the other degrees of freedom. There the system is described by an equation of motion (GLE) in which the dynamical effects of the environment appear as friction and random force. The friction in the GLE represents a memory effect in the dynamics in the sense that it depends on the values of the system variables in the past rather than just only their present values. The physical interpretation of this memory effect is that the present position of the environment, on which the force felt by the system depends, reflects an action from the system in the past. The GLE has provided us with several insights in understanding reactions in condensed phase. ${ }^{5,57-68}$

When the dynamics in the condensed phase is described by the GLE, a striking difference from gas phase arises from the fact that the system is always subject to external stochastic forces exerted by the environment. Even for a fixed initial condition of all the variables in the system, the final destination is not necessarily unique. Thus, concepts like the resonance overlap, ${ }^{69}$ based on the Hamiltonian formalism, or the deterministic area-preserving Poincaré map ${ }^{70}$ are not feasible for systems with dissipation and stochastic, external driving force that fluctuates thermally. To define an appropriate "TS" in a fluctuating media, some people have developed the socalled variational TS theory $(\mathrm{VTST})^{18,19}$ which optimizes a configurational "dividing" surface by minimizing the number of recrossings. The VTST has been applied with explicit harmonic bath modes. ${ }^{71-73}$ The VTST has provided excellent physical insights about the location of the dividing surface as well as improved expressions for rate constants. In practice, VTST needs a certain small number of selected variables to parametrise the dividing surface [usually position coordinate(s) of the system (e.g., solute)]. However, as indicated by Van der Zwan and Hynes ${ }^{74}$ and later by Pollak ${ }^{75}$ using a harmonic bath Hamiltonian system, the reaction coordinate must be, in principle, a function of all the position coordinates and velocities of the reacting system and all the effects exerted by the surroundings. It is far from trivial to identify which degrees of freedom are actually required to be involved.

Recent developments in reaction dynamics theories under the existence of thermally fluctuating environments $^{5,52,64-68,76-79}$ have made great progress in understanding the origins of reactions, that is, what type of initial conditions can bring the system to the reactant or the product. It was shown ${ }^{5,64-68}$ that, by introducing a coordinate shift for a given realization (i.e., time sequence) of the random force, the phase space structure dividing reactive and nonreactive trajectories can be extracted exactly in the case of a quadratic barrier without any nonlinear couplings among the modes. The theory has recently been generalized to a much wider class of systems in which multiple modes of the system generally interact with each other nonlinearly. ${ }^{52,76-79}$ Even with the existence of both the random force and nonlinear couplings among the modes, under the assumption of moderate magnitude of nonlinearity, a single coordinate still can be extracted which is decoupled from the others and its sign solely determines the destination region of the trajectory i.e., either the region of the reactant or the product. This new reaction coordinate is generally a nonlinear functional of all the position coordinates and velocities of the system plus the time-dependent external force.

When we know, or can reasonably assume, the statistical properties of the random force, we can calculate, for example, reaction rates ${ }^{57,58,66,78}$ from the statistics of the above-mentioned reaction coordinate, and the stochastic separatrix, ${ }^{63,77,80,81}$ which can be considered as the boundary between a phase space region with high reaction probability and that with low probability. Thus an equilibrium distribution of the bath is usually assumed when the GLE is used. ${ }^{56}$ An example of such a statistical property of the random force is the fluctuation-dissipation theorem, where the autocorrelation function of the random force is related to the friction kernel. The statistical property of the random force is derived by assuming a thermal equilibrium (or more generally, a stationary distribution) of the bath degrees of freedom. More precisely, the distribution is assumed to be a so-called constrained equilibrium, ${ }^{56}$ where the initial condition of the system can be specified arbitrarily but the distribution of the other modes 
(bath) is in equilibrium. However, one can often encounter many nonequilibrium molecular phenomena occurring with a non-stationary initial distribution of the bath. For example, in photo-excited reactions the initial distribution is determined by the response of the system to the light and thus can be different from the stationary one even along the coordinates other than the naïve "reaction coordinate." Sometimes one also excites the bath mode vibrations to obtain different reaction products. ${ }^{82-84}$ How we can acquire a low-dimensional description for such non-stationary systems has been an intriguing contemporary problem yet to be resolved.

For the last decade there have been several attempts at establishing the non-stationary representation termed the irreversible generalized Langevin equation (iGLE). ${ }^{85-94}$ In particular, the idea of generalized friction kernel ${ }^{88,94}$ dependent on time values in both the past and the present was proposed. As the (non-stationary) bath relaxes to the equilibrium state as time proceeds, the friction kernel is shown to converge to the usual equilibrium one, which depends only on the time difference. The generalization of the fluctuation-dissipation theorem was also proposed: the autocorrelation of the random force averaged over a given distribution of the initial condition of the bath degrees of freedom corresponds to the generalized time-dependent friction kernel ${ }^{88-94}$ or an exponentialdamping correction term, ${ }^{85-87}$ depending on how the iGLE is formulated. The iGLE can be applied not only to phenomena subject to an outside force that changes the solvent response, ${ }^{88}$ but also to systems whose non-stationarity is induced by the dynamics of the system itself. ${ }^{90}$ The growth of polymer was taken as an example where the property of the environment changes with the increase of the polymer length. It can also be applied to temperature-ramped chemical reactions ${ }^{91}$ where the environment temperature changes with time due to heating, for example, by a laser pulse. A further extension of iGLE $^{92}$ was provided to describe an ensemble of systems each of which is subject to a different random force and which are allowed to interact with each other.

In this article, we first review the GLE and its nonstationary extension in Sec. II. Then in Sec.III we discuss the dynamics in the saddle region in the framework of the Langevin equation, the memoryless limit of the GLE. The normal form theory for disentangling the effects of couplings will be introduced. In Sec. IV, we discuss the extension of the normal form theory into the GLE framework. The crux there is the introduction of variables that give an effective description of environmental motions. Finally, concluding remarks will be given in $\mathrm{Sec} \mathrm{V}$.

\section{GENERALIZED LANGEVIN EQUATION}

The nonlinear generalized Langevin Equation is given by ${ }^{55,56}$

$$
\frac{\mathrm{d}^{2}}{\mathrm{~d} t^{2}} q_{j}=f_{j}(\boldsymbol{q})-\int_{0}^{t} K_{j}\left(t-t^{\prime} ; \boldsymbol{q}\left(t^{\prime}\right), \dot{\boldsymbol{q}}\left(t^{\prime}\right)\right) \mathrm{d} t^{\prime}+\xi_{j}(t),
$$

where $\boldsymbol{q}=\left(q_{1}, q_{2}, \ldots, q_{n}\right)$ is a set of variables to describe the system, and $f_{j}(\boldsymbol{q})$ is called the mean force acting on the $j$ th variable, which is the average of the acceleration $\ddot{q}_{j}$ of the system for a fixed value of $\boldsymbol{q}$ over the probability distribution of the bath. When the mean force is obtained as a gradient of a certain function $U(\boldsymbol{q})$,

$$
f_{j}(\boldsymbol{q})=-\frac{\partial U(\boldsymbol{q})}{\partial q_{j}},
$$

the latter function is called the potential of mean force. The friction term $K_{j}\left(t-t^{\prime} ; \boldsymbol{q}\left(t^{\prime}\right), \dot{\boldsymbol{q}}\left(t^{\prime}\right)\right)$ depends on the history of the system. A possible physical interpretation of this term is that the bath is kicked by the system at time $t^{\prime}$ and this kick affects the bath configuration and its dynamics after time $t^{\prime}$. Then, at time $t$, the system feels a force from the bath that depends on the bath configuration at time $t$, the latter, in turn, depending on the kick in the past (at time $t^{\prime}$ ) by the system. If the bath is in a stationary distribution, this response of the bath only depends on the time difference, hence we have the $\operatorname{argument} t-t^{\prime}$ in $K$. The time-dependent force $\xi_{j}(t)$ represents another force from the bath that is purely determined by the initial condition of the bath. This third term is the so-called "random force", because we only observe the system and do not know the initial condition of the bath.

The friction term may be expanded in a polynomial form

$$
K_{j}\left(t-t^{\prime} ; \boldsymbol{q}\left(t^{\prime}\right), \dot{\boldsymbol{q}}\left(t^{\prime}\right)\right)=\sum_{\boldsymbol{m}} \gamma_{j \boldsymbol{m}}\left(t-t^{\prime}\right) \prod_{i=1}^{n} q_{i}\left(t^{\prime}\right)^{m_{i}} \dot{q}_{i}\left(t^{\prime}\right)^{m_{n+i}}
$$

$\left[\gamma_{j m}\left(t-t^{\prime}\right)\right.$ is an expansion coefficient depending on the time difference $t-t^{\prime}$ for each combination of powers $\boldsymbol{m}=$ $\left.\left(m_{1}, m_{2}, \ldots, m_{2 n}\right)\right]$, or in general by using a complete set of basis functions $\left\{\phi_{\ell}\right\}$ :

$$
K_{j}\left(t-t^{\prime} ; \boldsymbol{q}\left(t^{\prime}\right), \dot{\boldsymbol{q}}\left(t^{\prime}\right)\right)=\sum_{\ell} \gamma_{j \ell}\left(t-t^{\prime}\right) \phi_{\ell}\left(\boldsymbol{q}\left(t^{\prime}\right), \dot{\boldsymbol{q}}\left(t^{\prime}\right)\right) .
$$

Approximation of $K_{j}$ by truncating the summation and taking only the terms proportional to the velocity in these expansions yields

$$
K_{j}\left(t-t^{\prime} ; \boldsymbol{q}\left(t^{\prime}\right), \dot{\boldsymbol{q}}\left(t^{\prime}\right)\right) \approx \sum_{i} \gamma_{i j}\left(t-t^{\prime}\right) \dot{q}_{i}\left(t^{\prime}\right)
$$

giving the approximated GLE

$$
\frac{\mathrm{d}^{2}}{\mathrm{~d} t^{2}} q_{j}=f_{j}(\boldsymbol{q})-\int_{0}^{t} \sum_{i} \gamma_{i j}\left(t-t^{\prime}\right) \dot{q}_{i}\left(t^{\prime}\right) \mathrm{d} t^{\prime}+\xi_{j}(t) .
$$

This is probably the most commonly used form of the GLE. In this form of GLE, the coefficient $\gamma_{i j}\left(t-t^{\prime}\right)$, which is a function of the time difference $t-t^{\prime}$, is called the friction kernel. The friction kernel and the random force $\xi_{i}(t)$ are related by the fluctuation-dissipation theorem:

$$
\left\langle\xi_{i}(t) \xi_{j}\left(t^{\prime}\right)\right\rangle=k_{\mathrm{B}} T \gamma_{i j}\left(t-t^{\prime}\right)
$$

where the bracket denotes the ensemble average over a stationary distribution, $k_{\mathrm{B}}$ is the Boltzmann constant, and $T$ is the temperature. 
By using the technique of projection operator, ${ }^{54}$ it has been proved $^{55,56}$ that any Hamiltonian system can be projected onto its subsystem obeying the GLE in the form of Eq. (1), without any assumptions or approximations under a stationary distribution of the bath degrees of freedom. On the other hand, the form of Eq. (6) with the linear friction is shown ${ }^{95}$ to be exact, when the bath is a collection of harmonic oscillators and the coupling to the system is bilinear. Cortés et al. ${ }^{96}$ went further to show that, when the couplings are linear in the system coordinate $(\boldsymbol{q})$ but nonlinear in the bath coordinates, the system still obeys Eq. (6) up to the first order in the system-bath coupling strength.

When the time scale of the bath is much faster than that of the system, the friction kernel, which expresses the response of the environment to the motion of the system, may be approximated by

$$
\gamma_{i j}\left(t-t^{\prime}\right)=2 \gamma_{i j}^{0} \delta\left(t-t^{\prime}\right)
$$

where $\gamma_{i j}^{0}$ 's on the right hand side are constants, and $\delta\left(t-t^{\prime}\right)$ is Dirac's delta function. In this limit one obtains

$$
\frac{\mathrm{d}^{2}}{\mathrm{~d} t^{2}} q_{j}=f_{j}(\boldsymbol{q})-\sum_{i} \gamma_{i j}^{0} \dot{q}_{i}+\xi_{j}(t)
$$

which is called the Langevin equation, with the fluctuationdissipation theorem

$$
\left\langle\xi_{i}(t) \xi_{j}\left(t^{\prime}\right)\right\rangle=2 k_{\mathrm{B}} T \gamma_{i j}^{0} \delta\left(t-t^{\prime}\right),
$$

which means that the random force $\boldsymbol{\xi}(t)$ is a white noise.

While there is a general proof for the validity of the GLE [Eq. (1)], its usefulness hinges on our ability to predict, at least statistically, the realization of the random force. The fluctuation-dissipation theorem in the form of Eq. (7) concerns only with the ensemble average of the product of the random force over an equilibrium (or more in general, stationary) distribution. When the bath cannot be assumed to be in a stationary state, then we cannot even know the statistical property of the random force. To overcome this difficulty, one can resort to another formulation of the GLE for non-stationary bath $^{54,88-94,97}$ (here we write the one-dimensional case for the sake of simplicity):

$$
\frac{\mathrm{d}^{2}}{\mathrm{~d} t^{2}} q=f(q, \dot{q}, t)-\int_{0}^{t} \gamma\left(t, t^{\prime}\right) \dot{q}\left(t^{\prime}\right) \mathrm{d} t^{\prime}+\xi(t) .
$$

The key difference is the explicit dependence of the mean force on time $t$ and the dependence of the friction kernel $\gamma\left(t, t^{\prime}\right)$ on both the "initial" $\left(t^{\prime}\right)$ and the "final" $(t)$ times. In stationary state, the response of the bath to the motion of the system in the past depends only on the time difference $t-t^{\prime}$. Since the property of the bath is changing in time for the non-stationary case, the response of the bath depends on both times, and the mean force that the system feels also changes with time. In the non-stationary case, the fluctuation-dissipation theorem holds in the following form ${ }^{97}$ :

$$
\left\langle\xi(t) \xi\left(t^{\prime}\right)\right\rangle=\left\langle\dot{q}^{2}\right\rangle_{t^{\prime}} \gamma\left(t, t^{\prime}\right),
$$

where $\left\langle\dot{q}^{2}\right\rangle_{t^{\prime}}$ is the ensemble average of squared velocity at time $t^{\prime}$ over an initial distribution. The form of Eq. (11) is called the irreversible generalized Langevin equation (iGLE). ${ }^{88-94,97}$

While Eqs. (11) and (12) are proved ${ }^{97}$ to hold generally, Refs. 88-94 proposed a modeling such that the non-stationary random force is given by a time-dependent scaling of the equilibrium random force:

$$
\xi(t)=g(t) \xi^{\mathrm{eq}}(t)
$$

where the superscript 'eq' denotes the equilibrium state, and $g(t)$ is the scaling factor. Combining Eqs. (12) and (13), and considering the case of quasi-equilibrium ${ }^{88}$ between the system and the bath, $\left\langle\dot{q}^{2}\right\rangle_{t^{\prime}} \approx k_{\mathrm{B}} T_{\text {bath }}$, where $T_{\text {bath }}$ is the bath temperature, one sees that the friction kernel is also given by scaling:

$$
\gamma\left(t, t^{\prime}\right)=g(t) \gamma^{\mathrm{eq}}\left(t-t^{\prime}\right) g\left(t^{\prime}\right) .
$$

Ref. 94 introduced a more generalized model for the friction kernel allowing for multiple heat baths and time dilatation in the arguments of the equilibrium friction kernels:

$$
\gamma\left(t, t^{\prime}\right)=\sum_{k} \frac{T_{k}(t)}{T_{k}(0)} g_{k}(t) g_{k}\left(t^{\prime}\right) \gamma^{\mathrm{eq}(k)}\left(\tau_{k}(t)-\tau_{k}\left(t^{\prime}\right)\right),
$$

where $k$ labels each heat bath and $T_{k}$ is the time-dependent "temperature" of the $k$ th bath. The function $\tau_{k}(t)$ is a monotonically increasing function of $t$ whose rate of increase is time-dependent, reflecting the frequency modulation with time for each bath. Whereas the iGLE [Eq. (11)] and the non-stationary fluctuation-dissipation theorem [Eq. (12)] hold for any general non-stationary system ${ }^{97}$, Eqs. (13)-(15) were found to hold only for a special class of the total Hamiltonian system. $^{89,93}$

When we change the description of the system from the trajectory level to the distribution $\rho(\boldsymbol{q}, \dot{\boldsymbol{q}})$ level in the positionvelocity space, the Langevin equation (9) leads to the FokkerPlanck equation ${ }^{56}$

$$
\begin{aligned}
\frac{\partial \rho}{\partial t}= & -\sum_{j} \dot{q}_{j} \frac{\partial}{\partial q_{j}} \rho-\sum_{j} \frac{\partial}{\partial \dot{q}_{j}}\left(f_{j}(\boldsymbol{q})-\sum_{i} \gamma_{i j}^{0} \dot{q}_{i}\right) \rho \\
& +\sum_{i j} \gamma_{i j}^{0} k_{\mathrm{B}} T \frac{\partial^{2}}{\partial \dot{q}_{i} \partial \dot{q}_{j}} \rho .
\end{aligned}
$$

Kramers ${ }^{57}$ started with the Fokker-Planck equation of a onedimensional parabolic potential, and derived analytical expressions for barrier-crossing rates. In the low-friction regime, the rate-limiting step is the excitation in the well region, and the rate is proportional to the friction constant because the excitation is given by the random force, whose amplitude is related to the friction kernel through Eq. (10). On the other hand, in the moderate- to high-friction regimes, the rate is determined by the steady-state flow through the barrier region. There the rate is given by an eigenvalue of the linear dynamics at the saddle point, which becomes inversely proportional to the friction constant for high friction. Mel'nikov 
and Meshkov ${ }^{98}$ pursued this analysis of the rate constant under the assumption of high barrier height, and obtained a formula that connects those two regions. Their method is based on the use of an energy-action representation for writing the Fokker-Planck equation, which leads to the calculation of the steady-state flow with the activation rate in the well region correctly taken into account. The assumption of high barrier height compared with the temperature enabled a simple analytical expression for the Green function of the FokkerPlanck equation. Langer ${ }^{99}$ gave the multi-dimensional version of the rate formula in the moderate- to high-friction regimes, again with the parabolic approximation for the potential energy. The analysis proceeds similarly by obtaining the steady-state solution of the Fokker-Planck equation, and the rate is given by an eigenvalue of the linear dynamics in the multi-dimensional space. The assumption of high enough barrier made in the works cited above, in addition to facilitating the calculation, may ensure the existence of the rate, that is, single-exponential relaxation of the population, because then the distribution would quickly thermalize in the well region before crossing the barrier. One way to check whether the relaxation is actually single exponential is to calculate eigenvalues of the Fokker-Planck equation. If the lowest eigenvalue is real and much smaller than the others, the relaxation is then approximated by a single exponential kinetics with the rate equal to that eigenvalue. Voigtlaender and Risken ${ }^{100}$ used a matrix continued fractions method to calculate the eigenvalues of the Fokker-Planck equation for a one-dimensional double-well potential expressed by a quartic polynomial. It is shown that the lowest nonzero eigenvalue coincides with the Kramers formula in both the lowand high-friction regions, and smoothly connects them. The eigenfunction corresponding to the lowest eigenvalue spirals in the phase space in the opposite direction to the stochastic separatrix. Another way to check the exponential nature of the population relaxation is to calculate the number correlation function. Zhou ${ }^{101}$ adopted the latter method in a similar quartic potential. For high barrier height compared with the temperature, the relaxation is single-exponential, whereas for low barrier height it was found to be non-exponential as expected. Between these two regions, there exists a region where the relaxation is single-exponential but the rate is significantly different from the Mel'nikov-Meshkov formula. In the case of the GLE, where we have the memory effect, we can also move to the distribution-level description. The time evolution of the distribution is, however, much more complicated. ${ }^{56,102}$ The diffusion term involves integration over the physical variable as well as over the past time, reflecting the memory effect.

\section{SADDLE REGION DYNAMICS OF THE LANGEVIN EQUATION}

This section is aimed at the investigation of chemical reactions in the framework of the GLE and the iGLE presented in Sec. II. Specifically, we ask the following question: "Given an initial condition (i.e., the values of the position and the velocity of the reacting system at a certain time), can we pre- dict whether the system undergoes the reaction or not?" or, in other words, "What determines the fate of reactions in a thermally fluctuating environment?" When the system is parameterised by position coordinates $\boldsymbol{q}$, the reactant, which is the state before the reaction, and the product, which is the state after the reaction, correspond to certain stable regions in the $\boldsymbol{q}$-space, characterized by wells of the potential of mean force. In many cases, there is a saddle point on the potential between these two regions. The dynamics in the neighborhood of the saddle point plays a central (although not unequivocal) role in determining whether the system is going into the product region or back to the reactant region. We therefore concentrate our discussion in this section on the crossing dynamics in the neighborhood of the saddle point.

In Sec. III A, we start our discussion with a sufficiently simple case, the Langevin equation with a linear mean force and no random force. The effect of the random force is then incorporated in Sec. III B. The problem of the nonlinearity in the mean force, especially the couplings among the system coordinates, will be taken into account in Sec. III C. The structure of the dynamics is discussed in Sec. IIID based on the formulation presented in Secs. III A-III C. The extension to a generalized Langevin equation will be discussed in Sec. IV.

\section{A. Linear Dynamics in Saddle Region}

Let us start the discussion with a very simple case; a onedimensional Langevin equation where the mean force is linear in $q$ and we tentatively neglect the effect of the random force:

$$
\ddot{q}=\omega_{\mathrm{b}}^{2} q-\gamma \dot{q}
$$

where we changed the notation of the friction coefficient from $\gamma^{0}$ [see Eq. (9)] to $\gamma$. This mean force can be obtained as a derivative $-\partial U(q) / \partial q$ of an inverted parabolic potential with curvature $\omega_{\mathrm{b}}^{2}>0$

$$
U(q)=-\frac{1}{2} \omega_{\mathrm{b}}^{2} q^{2}
$$

Eq. (17) can be cast into matrix form

$$
\begin{aligned}
\frac{\mathrm{d}}{\mathrm{d} t}\left(\begin{array}{c}
q \\
\dot{q}
\end{array}\right) & =\mathbf{M}\left(\begin{array}{l}
q \\
\dot{q}
\end{array}\right), \\
\mathbf{M} & \stackrel{\operatorname{def}}{=}\left(\begin{array}{cc}
0 & 1 \\
\omega_{\mathrm{b}}{ }^{2} & -\gamma
\end{array}\right) .
\end{aligned}
$$

Following Ref. 63, we can introduce the eigenvectors of this matrix as

$$
\mathbf{M} \boldsymbol{v}_{ \pm}=\lambda_{ \pm} \boldsymbol{v}_{ \pm}
$$

where

$$
\begin{aligned}
& \lambda_{ \pm}=\frac{1}{2}\left(-\gamma \pm \sqrt{4 \omega_{\mathrm{b}}^{2}+\gamma^{2}}\right), \\
& \boldsymbol{v}_{ \pm}= \pm\left(\begin{array}{c}
1 \\
\lambda_{ \pm}
\end{array}\right) .
\end{aligned}
$$




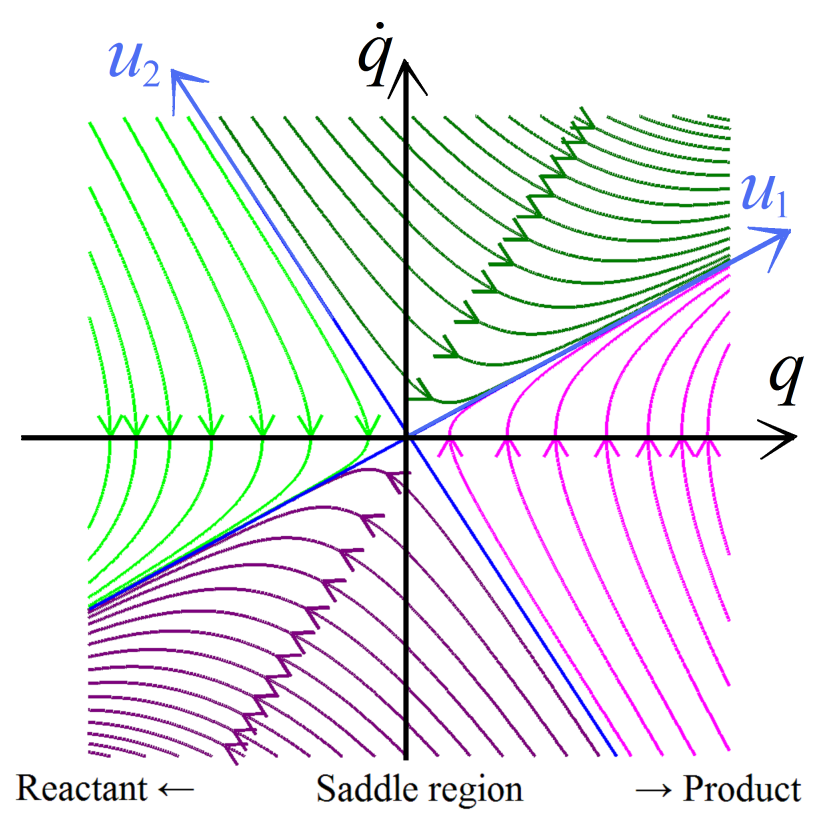

FIG. 1: Phase space flow in the $q-\dot{q}$ plane in the case of linear mean force without random force. It is seen that the trajectories with positive $u_{1}$ go eventually to the right side (the product), while those with negative $u_{1}$ go to the left side (the reactant).

Thus, by introducing a new coordinate system $\left(u_{1}, u_{2}\right)$ by

$$
\left(\begin{array}{c}
q \\
\dot{q}
\end{array}\right)=u_{1} \boldsymbol{v}_{+}+u_{2} \boldsymbol{v}_{-},
$$

the equations of motion become

$$
\begin{aligned}
\frac{\mathrm{d}}{\mathrm{d} t} u_{1} & =\lambda_{+} u_{1}, \\
\frac{\mathrm{d}}{\mathrm{d} t} u_{2} & =\lambda_{-} u_{2} .
\end{aligned}
$$

Since $\lambda_{+}$is a positive number, the absolute value of $u_{1}$ increases exponentially with time. This mode corresponds to a motion sliding down the barrier. On the other hand, the motion of $u_{2}$ (note $\lambda_{-}<0$ ) corresponds to a motion of approaching the barrier top and converging to it.

Figure 1 shows a picture of the phase space flow in the $(q, \dot{q})$-plane as well as the skewed coordinates $\left(u_{1}, u_{2}\right)$ introduced above. Suppose that the region with large negative $q$ is identified as the reactant region and that with large positive $q$ as the product. It is seen that the trajectories with $u_{1}>0$ go to the product region, while those with $u_{1}<0$ go to the reactant region. This figure has a good correspondence with the preceding studies on the Hamiltonian systems ${ }^{6,7,20-36}$ where the canonical conjugate pair $\left(\bar{q}_{1}, \bar{p}_{1}\right)$ was used as the "reaction coordinate." As stated above, the sign of $u_{1}$ can be used to discriminate the destination of the trajectory, that is, whether it is going to the product or returning back to the reactant. As soon as we just know the sign of $u_{1}$ in the saddle region, we can immediately tell, without further numerical propagation, whether the trajectory is going to the product region or to the reactant region.
Multi-dimensional cases can be treated similarly. Let us consider a multi-dimensional linear system

$$
\ddot{q}_{i}=\sum_{j} a_{i j} q_{j}-\sum_{j} \gamma_{i j} \dot{q}_{j},
$$

where the mean force is given by a linear combination of $\boldsymbol{q}=$ $\left(q_{1}, \ldots, q_{n}\right)$ with coefficients $a_{i j}$ and the friction is linear in $\dot{\boldsymbol{q}}$ with coefficients $\gamma_{i j}$. This can be written in a matrix form:

$$
\begin{aligned}
\frac{d}{d t}\left(\begin{array}{c}
\boldsymbol{q} \\
\dot{\boldsymbol{q}}
\end{array}\right) & =\mathbf{M}\left(\begin{array}{l}
\boldsymbol{q} \\
\dot{\boldsymbol{q}}
\end{array}\right), \\
\mathbf{M} & \stackrel{\text { def }}{=}\left(\begin{array}{cc}
\mathbf{0} & \mathbf{1} \\
\mathbf{A} & -\boldsymbol{\Gamma}
\end{array}\right),
\end{aligned}
$$

where $\mathbf{1}$ is the $n \times n$ unit matrix, $\mathbf{\Gamma}=\left(\gamma_{i j}\right)$ and $\mathbf{A}=\left(a_{i j}\right)$. The coordinate $\boldsymbol{u}=\left(u_{1}, u_{2}, \ldots, u_{2 n}\right)^{\mathrm{T}}$ is obtained by diagonalizing the matrix

$$
\begin{aligned}
& \mathbf{V}^{-1} \mathbf{M V}=\operatorname{diag}\left(\lambda_{1}, \cdots, \lambda_{2 n}\right), \\
& \left(\begin{array}{c}
\boldsymbol{q} \\
\dot{\boldsymbol{q}}
\end{array}\right)=\mathbf{V} \boldsymbol{u} .
\end{aligned}
$$

Here we can reasonably assume that there is one positive real eigenvalue $\lambda_{1}>0$ corresponding to the motion sliding down the barrier, and the others have $\operatorname{Re}\left(\lambda_{j}\right) \leq 0(j=2, \ldots, 2 n)$.

After the diagonalization, the equation of motion becomes

$$
\frac{d}{d t} u_{j}=\lambda_{j} u_{j}
$$

Since $u_{1}$ is the only unstable mode, just the knowledge of the sign of $u_{1}$ is sufficient to tell the destination region of trajectories. The surface defined by $u_{1}=0$ acts as an impenetrable boundary between reactive and non-reactive trajectories. The word 'impenetrable' here means that no trajectory can cross the boundary from $u_{1}>0$ to $u_{1}<0$ or vice versa, and, once the system is on that boundary, it remains there perpetually.

\section{B. Effect of Random Force - Time-dependent Shift of Origin}

Next we consider the effect of random force, but the mean force is still assumed to be linear in $\boldsymbol{q}$. The equation is given by

$$
\ddot{q}_{i}=\sum_{j} a_{i j} q_{j}-\sum_{j} \gamma_{i j} \dot{q}_{j}+\xi_{i}(t)
$$

with the fluctuation-dissipation relation

$$
\left\langle\xi_{i}(t) \xi_{j}(0)\right\rangle=2 k_{\mathrm{B}} T \gamma_{i j} \delta(t) .
$$

In matrix form,

$$
\frac{d}{d t}\left(\begin{array}{c}
\boldsymbol{q} \\
\dot{\boldsymbol{q}}
\end{array}\right)=\mathbf{M}\left(\begin{array}{c}
\boldsymbol{q} \\
\dot{\boldsymbol{q}}
\end{array}\right)+\left(\begin{array}{c}
\mathbf{0} \\
\boldsymbol{\xi}(t)
\end{array}\right),
$$

with the same matrix $\mathbf{M}$ as Eq. (25). By introducing the eigenmodes in the same way as Eq. (26), we obtain

$$
\frac{d}{d t} u_{j}=\lambda_{j} u_{j}+\tilde{\xi}_{j}(t)
$$


where $\tilde{\boldsymbol{\xi}}(t)=\mathbf{V}\left(\begin{array}{c}\mathbf{0} \\ \boldsymbol{\xi}(t)\end{array}\right)$.

To consider the dynamical origin of the reactions that are subject to the random force, a set of new relative coordinates, which we call $x_{j}$ here, was introduced recently: ${ }^{5,64-68}$

$$
u_{j}=S\left[\lambda_{j}, \tilde{\xi}_{j}\right](t)+x_{j}
$$

where we use the notation $S$ as in Refs. 5,52,76-79,103,104:

$$
S\left[\lambda_{j}, \tilde{\xi}_{j}\right](t)= \begin{cases}\int_{-\infty}^{0} \exp \left(-\lambda_{j} \tau\right) \tilde{\xi}_{j}(t+\tau) d \tau & \left(\operatorname{Re} \lambda_{j}<0\right) \\ -\int_{0}^{+\infty} \exp \left(-\lambda_{j} \tau\right) \tilde{\xi}_{j}(t+\tau) d \tau & \left(\operatorname{Re} \lambda_{j}>0\right)\end{cases}
$$

Then the equation of motion for $\boldsymbol{x}$ is given by

$$
\frac{d}{d t} x_{j}=\lambda_{j} x_{j}
$$

In the shifted coordinate system $\boldsymbol{x}$, the equation of motion for $x_{j}$ does not depend on the random force any more. As in Sec. III A, we assume that one and only one eigenvalue $\lambda_{1}$ has positive real part. Therefore the system would move away from the barrier region as $t$ increases, with the direction determined solely by the sign of $x_{1}$. Note that, in the definition of Eq. (33), it is necessary to know the time evolution of $\xi_{j}(t)$ for each instance in advance in order to calculate the shift. Therefore, Eq. (34) is formally correct, but in practice we need to replace this hypothetical requirement by the statistical properties of the random force, which will be discussed in Sec. III D.

\section{Effects of Nonlinearity - Normal Form Theory}

In realistic situations, the system is often subject to nonlinearity in the force. As we will see later in this section, the nonlinearity induces couplings among the shifted coordinates $x_{j}$. Then the motion of $x_{1}$ is subject to the other modes as well as the random external force, which prevents us from predicting the destination of the reaction independently from $x_{2}, x_{3}, \ldots$. However, we will see that, by introducing a nonlinear transformation, we can still extract a single reaction coordinate that enables us to predict the fate of the reaction without referring to the other coordinates. ${ }^{52,76-78}$

The nonlinear force can be expanded in a power series as

$$
\begin{aligned}
\ddot{q}_{i}= & \sum_{j} a_{i j} q_{j}+\sum_{|\boldsymbol{m}| \geq 2} \alpha_{j, \boldsymbol{m}} q_{1}^{m_{1}} q_{2}^{m_{2}} \cdots q_{n}^{m_{n}} \\
& -\sum_{j} \gamma_{i j} \dot{q}_{j}+\xi_{i}(t)
\end{aligned}
$$

with the expansion coefficients $\alpha_{j, \boldsymbol{m}}$ for each power $\boldsymbol{m}=$ $\left(m_{1}, m_{2}, \ldots, m_{n}\right)$. The nonlinear part contains terms of quadratic and higher order, that is, $|\boldsymbol{m}| \stackrel{\text { def }}{=} m_{1}+m_{2}+\cdots+m_{n} \geq 2$.

Introducing the linear transformation (Sec. III A) and the time-dependent shift (Sec. III B) the equation of motion be- comes

$$
\begin{aligned}
\frac{d}{d t} x_{j} & =\lambda_{j} x_{j}+f_{j}(\boldsymbol{x}, t), \\
f_{j}(\boldsymbol{x}, t) & =\sum_{|\boldsymbol{m}| \geq 0} f_{j, \boldsymbol{m}}(t) x_{1}^{m_{1}} x_{2}^{m_{2}} \cdots x_{2 n}^{m_{2 n}},
\end{aligned}
$$

where the coefficients $f_{j, m}(t)$ are obtained by substituting the transformation $\boldsymbol{q} \mapsto \boldsymbol{x}$ into the nonlinear part $\left(\sum_{|\boldsymbol{m}| \geq 2} \alpha_{j, \boldsymbol{m}} q_{1}^{m_{1}} \cdots q_{n}^{m_{n}}\right)$ of Eq. (35). The expansion coefficients $f_{j, m}(t)$ are now time-dependent because the transformation contains the time-dependent shift [Eq. (32)]. Due to the presence of the nonlinear terms, the dynamics of the reactive degree of freedom $x_{1}$ depends on all the other coordinates $x_{2}, x_{3}, \ldots, x_{2 n}$ as well as on all $\tilde{\xi}$ through the time dependence in $f_{j}(\boldsymbol{x}, t)$ that originates from that of the random force $\boldsymbol{\xi}(t)$.

To incorporate the effect of nonlinear terms in Eq. (36), we employ non-Hamiltonian normal form (NF) theory ${ }^{105}$ to introduce a nonlinear transformation from $\boldsymbol{x}$ to $\boldsymbol{y}$ so that the equation of motion for $y_{1}$ contains no coupling with the other coordinates. The calculation proceeds in a similar way to Lie canonical perturbation theory ${ }^{106,107}$ (a classical analog of Van Vleck perturbation theory). By the transformation

$$
x_{j}=y_{j}+w_{j}(\boldsymbol{y}, t)
$$

the equation of motion in $\boldsymbol{x}$ [Eq. (36)] is cast into

$$
\frac{d}{d t} y_{j}=\lambda_{j} y_{j}+c_{j}(\boldsymbol{y}, t)
$$

where the two unknown functions $\left(c_{j}\right.$ and $\left.w_{j}\right)$ are to be determined so that the resultant equations of motion for the new coordinate $\boldsymbol{y}$ [Eq. (38)] are in as simple a form as possible, and the transformation [Eq. (37)] has a good convergence at the same time.

Expanding the two functions in power series

$$
\begin{aligned}
& c_{j}(\boldsymbol{y}, t)=\sum_{\boldsymbol{m}} c_{j, \boldsymbol{m}}(t) y_{1}{ }^{m_{1}} \cdots y_{2 n}{ }^{m_{2 n}}, \\
& w_{j}(\boldsymbol{y}, t)=\sum_{\boldsymbol{m}} w_{j, \boldsymbol{m}}(t) y_{1}^{m_{1}} \cdots y_{2 n}^{m_{2 n}},
\end{aligned}
$$

and performing some involved algebra, ${ }^{76}$ we arrive at an equation of the following form:

$$
\left(\langle\lambda, \boldsymbol{m}\rangle-\lambda_{j}+\frac{d}{d t}\right) w_{j, \boldsymbol{m}}(t)=g_{j, \boldsymbol{m}}(t)-c_{j, \boldsymbol{m}}(t),
$$

where $\langle\lambda, \boldsymbol{m}\rangle \stackrel{\text { def }}{=} \sum_{k=1}^{2 n} \lambda_{k} m_{k}$ and $g_{j, \boldsymbol{m}}(t)$ are the coefficients of the polynomial expansion of $g_{j}(\boldsymbol{y}, t) \stackrel{\text { def }}{=} f_{j}(\boldsymbol{y}+\boldsymbol{w}, t)-$ $\sum_{i=1}^{2 n} c_{i}(\boldsymbol{y}, t) \frac{\partial w_{j}(\boldsymbol{y}, t)}{\partial y_{i}}$. As is shown in a previous paper, ${ }^{76}$ in the process of determining $c_{j, m}(t)$ and $w_{j, m}(t)$ perturbationally order by order, $g_{j, m}(t)$ is known from the results of the lower orders. There thus exist two unknown quantities $c_{j, m}(t)$ and $w_{j, m}(t)$ with the known $g_{j, m}(t)$ at each order. One can then determine either of the two unknown quantities as one wishes. To simplify the equation of motion for the new variable $y_{1}$, one can eliminate $c_{1, m}(t)$ order by order by setting

$$
w_{1, \boldsymbol{m}}(t)=S\left[\lambda_{1}-\langle\lambda, \boldsymbol{m}\rangle, g_{1, \boldsymbol{m}}\right](t),
$$


using the $S$-symbol as in Eqs. (32) and (33). The variable $y_{1}$ obeying a simplified equation of motion can then be regarded as a new reactive mode coordinate, while all the effects of nonlinear couplings, friction and random forces are incorporated in the transformation from the original coordinates $\boldsymbol{q}$ to $\boldsymbol{y}$.

In order to simplify the equation of motion for $\boldsymbol{y}$, making $c_{j, m}(t)=0$ may be the best choice. However, if we try to cancel as many $c_{j, m}(t)$ 's as possible by Eq. (40), the transformation $\left[w_{j}(\boldsymbol{y}, t)\right]$ contains more and more terms, which possibly causes divergence in the series expansion of the transformation. In order to make a well-behaved transformation, therefore, we keep some terms in the equation of motion for $\boldsymbol{y}$ and yet make the equation easily solvable to investigate the dynamics. More precisely, we have two choices ${ }^{52,53,76}$ for the final form of the equation of motion for $y$ that result in different convergence properties. In the next section we look into these two choices and their dynamical structures.

\section{Two types of normal form}

\section{1. partial normal form}

For the purpose of telling the fate of the reaction, we need only to know the motion along the unstable direction $y_{1}$ which corresponds to the motion sliding down the barrier. Thus we can make the equation of $\dot{y}_{1}$ decoupled from the others, while leaving other modes as they are:

$$
\begin{aligned}
\dot{y}_{1} & =\left[\lambda_{1}+c_{1}(t)\right] y_{1}+O\left(\varepsilon^{N+1}\right) \\
\dot{y}_{2} & =f_{2}(\boldsymbol{y}) \\
\dot{y}_{3} & =f_{3}(\boldsymbol{y}) \\
& \vdots \\
\dot{y}_{2 n} & =f_{2 n}(\boldsymbol{y}) .
\end{aligned}
$$

We call this 'partial normal form,' in contrast to the 'full normal form' where the equations of motion for all the modes are made independent and the motion of $y_{j}$ depends only on $y_{j}$ for all $j=1,2, \ldots, 2 n$. In the case of the neighborhood of the rank-one saddle point, where we have only one eigenvalue with positive real part, it was found ${ }^{76}$ that the full normal form is impossible to construct, in contrast to Hamiltonian systems in which the full normal form can exist in a certain range of total energy above the saddle. $6,7,20-39,45,46$

From Eq. (42), the solution for the motion of $y_{1}$ is given by

$$
y_{1}(t)=y_{1}\left(t_{0}\right) \exp \left[\int_{t_{0}}^{t}\left(\lambda_{1}+c_{1}\left(t^{\prime}\right)\right) d t^{\prime}\right]+O\left(\varepsilon^{N+1}\right),
$$

where $t_{0}$ is a certain time giving the initial condition for $y_{1}$. Here, $\lambda_{1}$ comes from the linear approximation and the other term $c_{1}(t)$ originates from the effect of nonlinearity, which begins with a first-order perturbation term.

Of particular importance is the behavior as $t \rightarrow+\infty$. The exponent in Eq. (43) goes to infinity as $t \rightarrow+\infty$ if the longtime average of the perturbative terms in $c_{1}(t)$ is less than $\lambda_{1}$, which can usually be expected to hold. Then we have

$$
\lim _{t \rightarrow+\infty} y_{1}(t)= \begin{cases}+\infty & \left(y_{1}\left(t_{0}\right)>0\right) \\ -\infty & \left(y_{1}\left(t_{0}\right)<0\right)\end{cases}
$$

The increase of $\left|y_{1}(t)\right|$ means that the system departs from the vicinity of the saddle point, going either to the product side or to the reactant side. The direction of this departing motion is determined solely by the sign of $y_{1}$ at any time $t_{0}$.

The most important consequence of the partial normal form is that the motion along the $y_{1}$ mode is independent of the other modes $\left(y_{2}, \ldots, y_{2 n}\right)$. Since $y_{1}$ is the only relevant variable to tell the destination of the reaction, we can describe the process of chemical reaction with a single dynamical variable $y_{1}$, which we now call the "reaction coordinate". As a consequence of this independence of $y_{1}$, just the knowledge of the sign of $y_{1}$ at any one moment in the saddle region is sufficient to tell the fate of the reaction.

\section{2. minimal normal form}

Another type of normal form has been recognized recently. ${ }^{52,53}$ This is termed here as 'minimal normal form,' and ensures the existence of an impenetrable boundary between the reactive and nonreactive trajectories, even when the nonlinear couplings and the random force are significantly so large that obtaining the reaction coordinate independent of the other coordinates is not possible. The equation of motion after the transformation is given as

$$
\begin{aligned}
\dot{y}_{1} & =\left[\lambda_{1}+\tilde{c}_{1}(\boldsymbol{y}, t)\right] y_{1}+O\left(\varepsilon^{N+1}\right) \\
\dot{y}_{2} & =f_{2}(\boldsymbol{y}) \\
\dot{y}_{3} & =f_{3}(\boldsymbol{y}) \\
& \vdots \\
\dot{y}_{2 n} & =f_{2 n}(\boldsymbol{y}),
\end{aligned}
$$

where $\tilde{c}_{1}$ can now be a function of $\boldsymbol{y}$ in addition to time $t$. In other words, we keep in the equation of $\dot{y}_{1}$ all the terms that are linear or higher order of $y_{1}$. Only the terms that do not contain $y_{1}$ are removed by the transformation. The solution is formally given by

$$
y_{1}(t)=y_{1}\left(t_{0}\right) \exp \left[\int_{t_{0}}^{t}\left(\lambda_{1}+\tilde{c}_{1}\left(\boldsymbol{y}\left(t^{\prime}\right), t^{\prime}\right)\right) d t^{\prime}\right] .
$$

This solution is only formal in the sense that the right hand side contains $\boldsymbol{y}\left(t^{\prime}\right)$ which would have to be solved according to the very same equation of motion [Eq. (45)]. However, the important point is that $y_{1}=0$ forms an invariant set just as in the case of partial normal form (i.e., $d y_{1} / d t=0$ if $y_{1}=0$ ). This implies that once the system is in the manifold of $y_{1}=0$, the system should remain to reside there for $t \rightarrow \pm \infty$. In other words, no trajectory can cross the manifold $y_{1}=0$ from the region $y_{1}>0$ to $y_{1}<0$, or vice versa. Consequently, once we know the sign of $y_{1}\left(t_{0}\right)$ at any instant $t_{0}$ in the saddle region, it fixes the sign of $y_{1}$ for $t \rightarrow \infty$, telling us the final destination of 
the reaction. The minimal normal form was shown $n^{52,53}$ to have better convergence property because it contains less terms in the transformation.

Figure 2 shows schematic pictures for the flow of trajectories in the extended phase space. When there is no (or negligible) random force or nonlinearity, the normal mode reaction coordinate $u_{1}$ introduced as in Fig. 1 [see Sec. III A] obeys the simple equation [Eq. (23)] and the trajectories plotted in the $\left(t, u_{1}\right)$-plane follow exponential curves [Fig. 2 (a)]. Since the equation of motion for $u_{1}$ is independent of the other coordinates $u_{2}, \ldots, u_{2 n}$, the time propagation of $u_{1}$ is uniquely determined once the value of $u_{1}$ at a certain time $t$ is specified. This is why no two trajectories can cross with each other in Fig. 2 (a). The invariant set $\left\{(\boldsymbol{q}, \dot{\boldsymbol{q}}) \mid u_{1}=0\right\}$, or denoted simply by $\left\{u_{1}=0\right\}$, serves as an impenetrable reactivity boundary that separates trajectories going to the product side and those going to the reactant side. When the random force and the nonlinearity are considered, $u_{1}$ is no longer an independent coordinate. Even if we specify the values of $u_{1}$ and $t$ for a given realization of the random force $\xi(t)$, the future of the trajectory can differ according to the values of the other coordinates due to the couplings. The situation is plotted in Fig. 2 (b). Several trajectories cross each other in the $\left(t, u_{1}\right)$ plane. The set $\left\{u_{1}=0\right\}$ is no longer invariant and cannot tell us anything about the destination of the reaction. In a certain range of the magnitude of the random force and the nonlinearity, the remainder of the partial normal form [denoted by $O\left(\varepsilon^{N+1}\right)$ in Eq. (42)] can be made sufficiently small by proceeding with the calculation up to a certain order $N$. Then the equation of motion given by Eq. (42) gives a good approximate description of the dynamics of the system. In this case, the structure of the dynamics can be best captured by the plot with the partial normal form reaction coordinate $y_{1}^{\mathrm{p}}$ rather than $u_{1}$. The trajectories plotted in the $\left(t, y_{1}^{\mathrm{p}}\right)$-space exhibit a laminar flow as in Fig. 2 (c). Here 'laminar' means that no two trajectories can cross each other. This is because the motion of $y_{1}^{\mathrm{p}}$ is independent of the others in the partial normal form [Eq. (42)]. Specifying the value of $\left(t, y_{1}^{\mathrm{p}}\right)$ at any point is sufficient to tell the future time evolution of $y_{1}^{\mathrm{p}}$. In contrast to Fig. 2 (a), the time evolution of $y_{1}^{\mathrm{p}}$ is not exactly an exponential function because of the existence of $c_{1}(t)$ in Eq. (43). As the random force and the nonlinearity become larger, the partial normal form may not be convergent and then we can no longer extract an independent reaction coordinate. It is still possible that the minimal normal form gives a good approximation, while the partial normal form does not. Figure 2 (d) shows a phase space flow schematically for the case where the minimal normal form gives an appropriate description of the system. Due to the coupling between the minimal normal form reaction coordinate $y_{1}^{\mathrm{m}}$ and the others, the time evolution of $y_{1}^{\mathrm{m}}$ is not unique even for the same values of $\left(t, y_{1}^{\mathrm{m}}\right)$ at a certain point. Thus crossings among the trajectories are seen in the plot of trajectories in the $\left(t, y_{1}^{\mathrm{m}}\right)$-space. However, the set $\left\{y_{1}^{\mathrm{m}}=0\right\}$ is an invariant manifold as was discussed in Sec. III D 2. This means that no trajectories can cross the set $\left\{y_{1}^{\mathrm{m}}=0\right\}$ from one side to the other. One can therefore tell the destination of the trajectory once the sign of $y_{1}^{\mathrm{m}}$ is specified at a certain time.
In summary, we have introduced a new reaction coordinate $y_{1}$, that is, $y_{1}^{\mathrm{p}}$ or $y_{1}^{\mathrm{m}}$, through a transformation that incorporates all the effects of the random force and the nonlinear couplings. In both the partial and minimal normal forms, the sign of $y_{1}$ solely determines the destination of the reaction. The difference in the two normal forms is that, as seen in Fig. 2, the partial normal form is designed to have the reaction coordinate $y_{1}^{\mathrm{p}}$ decoupled from all the others, whereas the minimal normal form ensures only the existence of the reactivity boundary $y_{1}^{\mathrm{m}}=0$, and not the independence of the reaction coordinate $y_{1}^{\mathrm{m}}$ from the others. It was found ${ }^{52,53}$ that the minimal normal form has a better convergence in the perturbation theory than the partial normal form, implying that the impenetrable boundary persists to exist up to a higher temperature (i.e., higher energy) region although the concept of the reaction coordinate decoupled from the other coordinates is no longer applicable there.

The transformation constructed above to obtain the reaction coordinate $y_{1}$ depends on the random force. When we fix the instance of the random force, the transformation is fixed and the sign of $y_{1}$ tells us the fate of the reaction uniquely. In the case we consider the ensemble of the random force, which is more realistic, we have statistics for $y_{1}$ corresponding to the distribution of the random force. The reaction probability is then given as the probability to have $y_{1}>0$, for which the analytical formula was also given. ${ }^{78}$ For given values of $\boldsymbol{q}$ and $\dot{\boldsymbol{q}}$, we can calculate the average value $\left\langle y_{1}\right\rangle$ of $y_{1}$ over the distribution of the random force. Roughly, if $\left\langle y_{1}\right\rangle>0$, there is larger probability for the system to go into the product region than if $\left\langle y_{1}\right\rangle<0$. The curve given by $\left\langle y_{1}\right\rangle=0$ in the $(\boldsymbol{q}, \dot{\boldsymbol{q}})$ space can thus be regarded as the boundary between the region with higher reaction probability and that with lower probability. Note that $\left\langle y_{1}\right\rangle=0$ implies the reaction probability being exactly one half if the probability distribution of $y_{1}$ is Gaussian, but not in general. A physical interpretation about what makes the reaction happen can also be obtained by expressing the reaction coordinate $y_{1}$ in terms of the original coordinates $\boldsymbol{q}$, the velocity $\dot{\boldsymbol{q}}$ and the random force $\boldsymbol{\xi}(t)$. In Refs. 76 and 78 , the effects were classified as a direct effect of the environment, effects of nonlinearity, and combined effects of these two.

\section{FROM GLE TO MEMORYLESS EQUATIONS OF MOTION : EFFECTIVELY EXPRESSED ENVIRONMENTAL MODES}

A peculiar feature of the GLE, compared to the Langevin equation, is that it contains the memory term. This formally prohibits the direct application of the normal form theory presented in Sec. III to the GLE. In order to discuss the dynamics and phase space structures of the GLE, there have been several attempts ${ }^{60,63,65,67,79}$ to derive an equivalent memoryless equation of motion from the GLE. Here we follow a procedure that is basically adopted from Ref. 63. We treat mainly the case of linear friction [Eq. (6)], and the generalization to the nonlinear friction case [Eq. (1)] will be discussed later. We also confine 
(a)

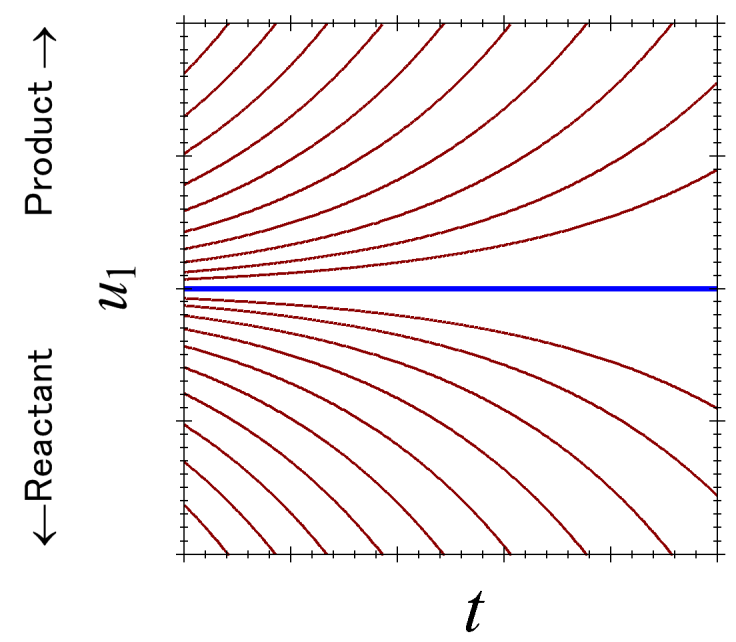

(c)

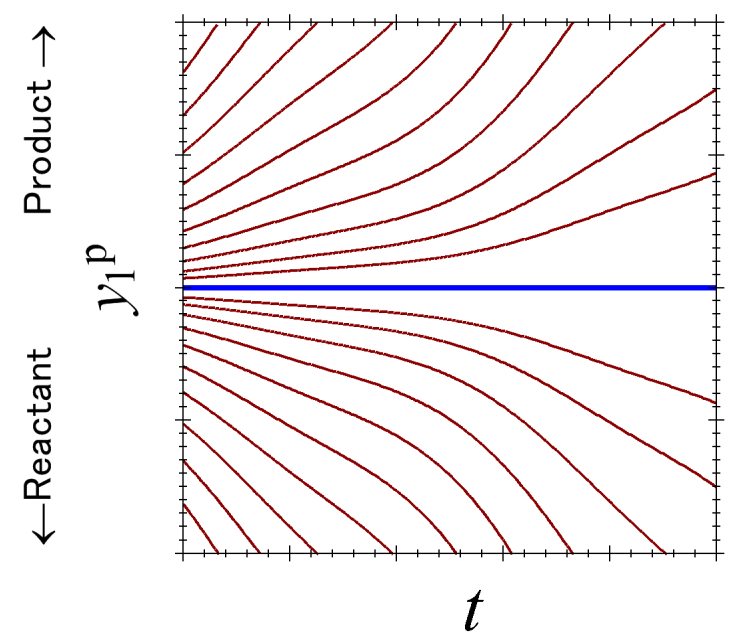

(b)

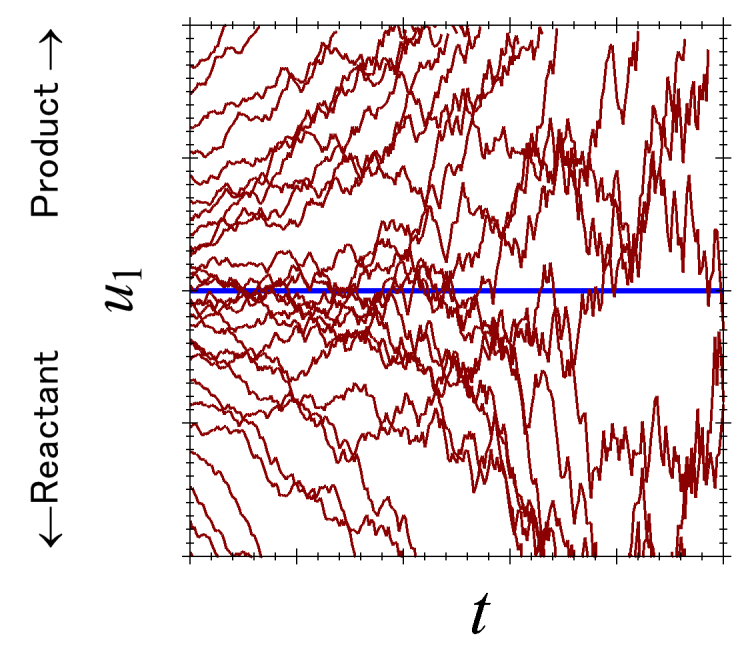

(d)

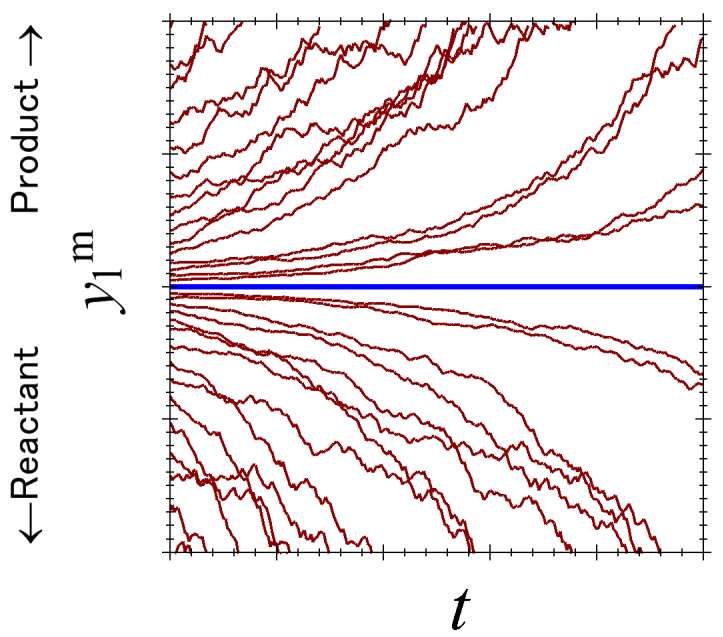

FIG. 2: Schematic pictures for the flow of trajectories in several regimes plotted in various coordinates and time. (a) When the random force and the nonlinearity is negligible, the trajectories plotted in $\left(t, u_{1}\right)$ follow simple exponential curves. (b) Under the effect of the random force and the nonlinearity, the time propagation of $u_{1}$ is no longer as simple as in (a). (c) For a range of the magnitudes of the random force and the nonlinearity, the partial normal form transformation provides a much better description of the system dynamics. The time propagation of the coordinate $y_{1}^{\mathrm{p}}$ shows a laminar flow. There is no crossing between any two trajectories. The impenetrable boundary $y_{1}^{\mathrm{p}}=0$ separates the trajectories going to the product region and those going to the reactant region. (d) For yet stronger random force and nonlinearity, it is no longer possible to construct an independent reaction coordinate. The reactivity boundary can still exist and be given by $y_{1}^{\mathrm{m}}=0$ in terms of the minimal normal form coordinate. The existence of crossings among trajectories shows the dependence of $\dot{y}_{1}^{\mathrm{m}}$ on the other coordinates.

our attention to the one-dimensional case

$$
\frac{\mathrm{d}^{2}}{\mathrm{~d} t^{2}} q=f(q)-\int_{0}^{t} \gamma\left(t-t^{\prime}\right) \dot{q}\left(t^{\prime}\right) \mathrm{d} t^{\prime}+\xi(t),
$$

for simplicity, and the extension to the multi-dimensional cases is straightforward. First we express the friction kernel in a multi-exponential function:

$$
\gamma(\tau)=\sum_{k} a_{k} \exp \left(-\mu_{k} \tau\right)
$$

The amplitude $a_{k}$ and the time constant $\mu_{k}$ may be obtained by fitting ${ }^{108,109}$ the given friction kernel to the form of Eq. (48).
These parameters can be complex-valued in the case where the friction kernel is an exponentially decaying oscillatory function. We then define new variables $\left\{\zeta_{k}\right\}$ by

$$
\zeta_{k} \stackrel{\text { def }}{=} \int_{0}^{t} a_{k} \exp \left(-\mu_{k}\left(t-t^{\prime}\right)\right) \dot{q}\left(t^{\prime}\right) \mathrm{d} t^{\prime}
$$


Then the GLE (47) is equivalent to the following set of equations

$$
\begin{aligned}
\frac{\mathrm{d}^{2}}{\mathrm{~d} t^{2}} q & =f(q)-\sum_{k} \zeta_{k}+\xi(t), \\
\frac{\mathrm{d}}{\mathrm{d} t} \zeta_{k} & =-\mu_{k} \zeta_{k}+a_{k} \dot{q},
\end{aligned}
$$

with the initial condition $\zeta_{k}=0$.

By expanding the $f(q)$ as $b q+\sum_{m>1} \alpha_{m} q^{m}$ and introducing vector notation as in Eq. (25),

$$
\begin{aligned}
\frac{\mathrm{d}}{\mathrm{d} t}\left(\begin{array}{c}
q \\
\dot{q} \\
\zeta_{1} \\
\vdots \\
\zeta_{K}
\end{array}\right) & =\left(\begin{array}{ccccc}
0 & 1 & 0 & \cdots & 0 \\
b & 0 & -1 & \cdots & -1 \\
0 & a_{1} & -\mu_{1} & \cdots & 0 \\
\vdots & \vdots & \vdots & \ddots & \vdots \\
0 & a_{K} & 0 & \cdots & -\mu_{K}
\end{array}\right)\left(\begin{array}{c}
q \\
\dot{q} \\
\zeta_{1} \\
\vdots \\
\zeta_{K}
\end{array}\right) \\
& +\left(\begin{array}{c}
\sum_{m>1} \alpha_{m} q^{m}+\xi(t) \\
0 \\
\vdots \\
0
\end{array}\right),
\end{aligned}
$$

where $K$ is the total number of exponential terms used in Eq. (48). Then, by diagonalizing the matrix in the first term and introducing the shift as in Sec. III B, we obtain equations in the form of Eq. (36), which can then be rendered into the normal form by the same procedure as presented in Sec. III C.

The generalization to the nonlinear friction case [Eq. (1)] may readily be obtained by the expansion in basis functions as in Eq. (4), and fitting each coefficient to multi-exponentials:

$$
\begin{aligned}
K\left(t-t^{\prime} ; q\left(t^{\prime}\right), \dot{q}\left(t^{\prime}\right)\right) & =\sum_{\ell} \gamma_{\ell}\left(t-t^{\prime}\right) \phi_{\ell}\left(q\left(t^{\prime}\right), \dot{q}\left(t^{\prime}\right)\right), \\
\gamma_{\ell}(\tau) & =\sum_{k} a_{\ell k} \exp \left(-\mu_{\ell k} \tau\right) .
\end{aligned}
$$

Then by defining $\zeta$ 's similarly to Eq. (49),

$$
\zeta_{\ell k} \stackrel{\text { def }}{=} \int_{0}^{t} a_{\ell k} \exp \left(-\mu_{\ell k}\left(t-t^{\prime}\right)\right) \phi_{\ell}\left(q\left(t^{\prime}\right), \dot{q}\left(t^{\prime}\right)\right) \mathrm{d} t^{\prime},
$$

the GLE [Eq. (1)] is equivalent to the following set of equations

$$
\begin{aligned}
\frac{\mathrm{d}^{2}}{\mathrm{~d} t^{2}} q & =f(q)-\sum_{\ell, k} \zeta_{\ell k}+\xi(t), \\
\frac{\mathrm{d}}{\mathrm{d} t} \zeta_{\ell k} & =-\mu_{\ell k} \zeta_{\ell k}+a_{\ell k} \phi_{\ell}(q, \dot{q}),
\end{aligned}
$$

with the initial condition $\zeta_{\ell k}=0$.

In our opinion, the above procedure is not only a mathematical tool to cast the GLE into a form that admits the application of NF theory, but we can also extract an interesting physical picture from it. Note that the friction term depending on the history of the system arises from dynamical interactions between the system and the environment. Here we use the word 'environment' to mean all the motions in the total system other than those described by the system variables $\boldsymbol{q}$. When $\boldsymbol{q}$ describe some limited number of structural variables (such as nuclear distances) of the solute, the 'environment' includes all the other degrees of freedom in the solute molecule as well as the motion of the solvent molecules. The friction term can be interpreted as a response of the environmental degrees of freedom to the system described by $\boldsymbol{q}$. More precisely, the system interacts with the environment, and then the configuration of the environment is changed due to the kick from the system. Then the force exerted by the environment to the system depends on the kick from the system accumulated over all the past times, giving rise to the memory term that contains the past values of the system variables. In the above procedure, we replaced the memory term by a set of extra variables. As such, the newly added variables can be interpreted as describing the environmental motions. In reality, the environmental motion may be a collection of atomic coordinates in the total system (the solute as well as the solvents). What we have done is to introduce some limited number of variables that effectively describe such collective motion in the environment, in a top-down way from the GLE obeyed by the system $\boldsymbol{q}$, rather than constructing them from the atomic potentials. Moreover, the friction kernel does not necessarily contain all the motions of the solvent molecules but includes those which are effectively coupled with the system. Therefore the above procedure is a way of extracting the effective finite degrees of freedom from the vast (practically infinite) dimensions of the environment.

The extension to the non-stationary case [Eq. (11)] can also be obtained. In this case, we fit the non-stationary friction kernel to the following form:

$$
\gamma\left(t, t^{\prime}\right)=\sum_{k} \varphi_{k}(t) \psi_{k}\left(t^{\prime}\right)
$$

Note that the multiexponential fit in the stationary case [Eq. (48)] can be regarded as a special case of Eq. (55), by putting

$$
\begin{aligned}
\varphi_{k}(t) & =a_{k} \exp \left(-\mu_{k} t\right), \\
\psi_{k}\left(t^{\prime}\right) & =\exp \left(\mu_{k} t^{\prime}\right) .
\end{aligned}
$$

The form of Eq. (15) proposed by Ref. 94 also falls into the form of Eq. (55), by first fitting the equilibrium kernel of each bath to multiexponential form

$$
\gamma_{i j}^{\mathrm{eq}(k)}\left(\tau_{k}(t)-\tau_{k}\left(t^{\prime}\right)\right)=\sum_{l} a_{i j}^{(k l)} \exp \left[-\mu_{i j}^{(k l)}\left\{\tau_{k}(t)-\tau_{k}\left(t^{\prime}\right)\right\}\right]
$$

and then setting

$$
\begin{aligned}
\varphi_{i j}^{(k l)}(t) & =\frac{T_{k}(t)}{T_{k}(0)} g_{k}(t) a_{i j}^{(k l)} \exp \left[-\mu_{i j}^{(k l)} \tau_{k}(t)\right], \\
\psi_{i j}^{(k l)}\left(t^{\prime}\right) & =g_{k}\left(t^{\prime}\right) \exp \left[\mu_{i j}^{(k l)} \tau_{k}\left(t^{\prime}\right)\right] .
\end{aligned}
$$

With the form of the non-stationary friction kernel given by 
Eq. (55), the iGLE becomes equivalent to

$$
\begin{aligned}
\frac{\mathrm{d}^{2}}{\mathrm{~d} t^{2}} q & =f(q, \dot{q}, t)-\sum_{k} \zeta_{k}+\xi(t), \\
\frac{\mathrm{d}}{\mathrm{d} t} \zeta_{k} & =\frac{\dot{\varphi}_{k}(t)}{\varphi_{k}(t)} \zeta_{k}+\varphi_{k}(t) \psi_{k}(t) \dot{q},
\end{aligned}
$$

where

$$
\zeta_{k} \stackrel{\text { def }}{=} \int_{0}^{t} \varphi_{k}(t) \psi_{k}\left(t^{\prime}\right) \dot{q}\left(t^{\prime}\right) \mathrm{d} t^{\prime}
$$

with the initial condition $\zeta_{k}=0$. Here we see that the time constant $\dot{\varphi}_{k}(t) / \varphi_{k}(t)$ of the environmental mode and the coupling strength $\varphi_{k}(t) \psi_{k}(t)$ are now time-dependent in contrast to the stationary case [Eq. (50)]. This implies that the timescales of environmental modes and the coupling strength between the environment and the system are modulated in time. We will provide some numerical examples elsewhere.

\section{SUMMARY AND OUTLOOK}

Including the solvent molecules, condensed phase chemical reactions involve a large (practically infinite) number of degrees of freedom. While, in principle, the reaction process can be described in a space taking the positions and momenta of all the atoms as coordinates (=practically infinite dimensional phase space), it is almost impossible to obtain physical insights in such a huge-dimensional space. One thus has to pick up a small number of variables that represent the progress of the reaction, usually some coordinates relevant to the reacting system and to project the equation of motion onto the GLE of those variables. Then the equation is no longer a Hamiltonian system and there arise two kinds of effects from the environment: friction and random force. The friction can exhibit a memory effect due to the response of the environment to the system, whereas the thermal fluctuation, the stochastic behaviour of the random force, arises from the fact that we do not know the initial condition of the environment (in the full phase space).

In Secs. II and IV of this article we have reviewed recent theoretical developments in the framework of several GLE formalisms and a new scheme was presented to transform the GLE in stationary and non-stationary environments into memoryless equations of motions. The information contained in the friction kernel was used to extract effective variables that represent the effects of the environment. The environmental variables introduced here do not describe all the details of the thermal reservoir but represent only those parts which contribute significantly to the motion of the variable(s) in consideration. Note that it is now possible to obtain the functional form of the friction kernel from $a b$ initio calculations ${ }^{110}$ and MD simulations. ${ }^{108,109,111}$ It is found there that the friction kernel can be fitted by a significantly limited number of terms compared to the infinitely many degrees of freedom of the solute plus the solvent. Thus a significant reduction of the description is possible by "summarizing" the motion of the environment. Comparing the results of these atomic level calculations ${ }^{108-111}$ and the environmental variables extracted in the present method would give significant insights into the condensed phase reaction dynamics by elucidating which degrees of freedom in the environment are able to affect the reacting system, what could characterize the aspects of the environment that can "kick" the system, and so forth.

After reducing the huge total system of the condensed phase reaction into a relatively low-dimensional dynamical system with the effective environmental variables, the equation of motion still contains couplings between the system variable(s) and these environmental modes, as well as the random force. Then the normal form procedure reviewed in Sec. III can take away these effects and extract a single reaction coordinate that is decoupled from all the rest. We can thus unveil what determines the fate of the reaction in "thermally" fluctuating environments.

The question of what role "thermal" fluctuation actually plays in allowing biological systems to function robustly is one of the most crucial, yet unresolved subjects in biology. Our theory can tell us the existence of an analytical expression for the reaction coordinate that guides the system to react as a nonlinear functional of the original position coordinates and the velocities, friction kernel, and random force. However, in most cases, one can know neither the equation of motion of biological systems nor the information of the full degrees of freedom of the total system. In experiments at single molecule level, one can monitor only one or a few observable(s). ${ }^{112} \mathrm{~A}$ crucial approach toward revealing the role of thermal fluctuation in the realm of biology would be to extract the underlying GLE or iGLE from observed single molecule time series and to look into the question of what reaction coordinate actually guides the biological system to react or function in a noisy environment by using the theoretical frameworks presented in this article.

\section{Acknowledgments}

This work has been supported by Research Fellowships of the Japan Society for the Promotion of Science for Young Scientists (to SK) and by JSPS, JST/CREST, Grant-in-Aid for Research on Priority Areas 'Molecular Theory for Real Systems' and 'Innovative Nanoscience' and a Human Frontier Science Program Research Grant (to TK). The computations were partially performed using the Research Center for Computational Science, Okazaki, Japan.
1 R. D. Levine, Molecular Reaction Dynamics, Cambridge University Press, Cambridge, 2005.
2 J. Zhang, D. Dai, C. C. Wang, S. A. Harich, X. Wang, X. Yang, 
M. Gustafsson and R. T. Skodje, Phys. Rev. Lett., 2006, 96, 093201.

${ }^{3}$ W. Shiu, J. J. Lin and K. Liu, Phys. Rev. Lett., 2004, 92, 103201.

${ }^{4}$ R. T. Skodje, D. Skouteris, D. E. Manolopoulos, S.-H. Lee, F. Dong and K. Liu, Phys. Rev. Lett., 2000, 85, 1206-1209.

5 T. Bartsch, J. M. Moix, R. Hernandez, S. Kawai and T. Uzer, $A d v$. Chem. Phys., 2008, 140, 191-238.

${ }^{6}$ T. Uzer, C. Jaffé, J. Palacián, P. Yanguas and S. Wiggins, Nonlinearity, 2002, 15, 957-992.

7 T. Komatsuzaki and R. S. Berry, J. Chem. Phys., 1999, 110, 9160-9173.

${ }^{8}$ C. Jaffe, S. D. Ross, M. W. Lo, J. Marsden, D. Farrelly and T. Uzer, Phys. Rev. Lett., 2002, 89, 011101.

9 C. P. Herrero and R. Ramírez, Phys. Rev. Lett., 2007, 99, 205504.

${ }^{10}$ M. Karplus, J. Phys. Chem. B, 2000, 104, 11-27.

11 P. J. Kuntz, E. M. Nemeth, J. C. Polanyi, S. D. Rosner and C. E. Young, J. Chem. Phys., 1966, 44, 1168-1184.

${ }^{12}$ H. Eyring, J. Chem. Phys., 1935, 3, 107-115.

13 E. Wigner, J. Chem. Phys., 1937, 5, 720-725.

${ }^{14}$ M. G. Evans and M. Polanyi, Trans. Faraday Soc., 1935, 31, 875-894.

15 O. K. Rice and H. C. Ramsperger, J. Am. Chem. Soc., 1928, 50, 617-620.

${ }^{16}$ L. S. Kassel, J. Phys. Chem., 1928, 32, 1065-1079.

17 R. A. Marcus, J. Chem. Phys., 1952, 20, 359-364.

18 J. C. Keck, Adv. Chem. Phys., 1967, 13, 85-121.

19 D. G. Truhlar and B. C. Garrett, Acc. Chem. Res., 1980, 13, 440448.

20 T. Komatsuzaki and M. Nagaoka, J. Chem. Phys., 1996, 105, 10838-10848.

21 T. Komatsuzaki and M. Nagaoka, Chem. Phys. Lett., 1997, 265, 91-98.

22 T. Komatsuzaki and R. S. Berry, Phys. Chem. Chem. Phys., 1999, 1, 1387-1397.

23 T. Komatsuzaki and R. S. Berry, J. Chem. Phys., 2001, 115, 4105-4117.

${ }^{24}$ T. Komatsuzaki and R. S. Berry, J. Mol. Struct. (Theochem), 2000, 506, 55-70.

25 T. Komatsuzaki and R. S. Berry, Proc. Nat. Acad. Sci. USA, 2001, 98, 7666-7671.

26 T. Komatsuzaki and R. S. Berry, J. Phys. Chem. A, 2002, 106, 10945-10950.

27 S. Wiggins, L. Wiesenfeld, C. Jaffé and T. Uzer, Phys. Rev. Lett., 2001, 86, 5478-5481.

${ }^{28}$ H. Waalkens, A. Burbanks and S. Wiggins, J. Chem. Phys., 2004, 121, 6207-6225.

${ }^{29}$ H. Waalkens, A. Burbanks and S. Wiggins, J. Phys. A, 2004, 37, L257-L265.

${ }^{30}$ H. Waalkens and S. Wiggins, J. Phys. A, 2004, 37, L435-L445.

${ }^{31}$ S. Kawai, Y. Fujimura, O. Kajimoto, T. Yamashita, C.-B. Li, T. Komatsuzaki and M. Toda, Phys. Rev. A, 2007, 75, 022714.

32 S. Kawai, C. Jaffé and T. Uzer, J. Phys. B: At. Mol. Opt. Phys., 2005, 38, S261-S278.

${ }^{33}$ C.-B. Li, Y. Matsunaga, M. Toda and T. Komatsuzaki, J. Chem. Phys., 2005, 123, 184301.

${ }^{34}$ C.-B. Li, A. Shojiguchi, M. Toda and T. Komatsuzaki, Few-Body Systems, 2006, 38, 173-179.

35 C.-B. Li, A. Shojiguchi, M. Toda and T. Komatsuzaki, Phys. Rev. Lett., 2006, 97, 028302.

${ }^{36}$ C.-B. Li, M. Toda and T. Komatsuzaki, J. Chem. Phys., 2009, 130, 124116.

37 Geometrical Structures of Phase Space in Multidimensional Chaos: Applications to Chemical Reaction Dynamics in Complex Systems, Adv. Chem. Phys., ed. M. Toda, T. Komatsuzaki,
T. Konishi, R. S. Berry and S. A. Rice, John-Wiley \& Sons, Inc., 2005, vol. 130A,130B.

38 Advancing Theory for Kinetics and Dynamics of Complex, ManyDimensional Systems: Clusters and Proteins, Adv. Chem. Phys., ed. T. Komatsuzaki, R. S. Berry and D. M. Leitner, John-Wiley \& Sons, Inc., 2011, vol. 145.

39 S. Kawai, H. Teramoto, C.-B. Li, T. Komatsuzaki and M. Toda, Adv. Chem. Phys., 2011, 145, 123-169.

${ }^{40}$ E. R. Lovejoy, S. K. Kim and C. B. Moore, Science, 1992, 256, 1541-1544.

${ }^{41}$ E. R. Lovejoy and C. B. Moore, J. Chem. Phys., 1993, 98, 78467854.

42 W. H. Miller, Faraday Discussions Chem. Soc., 1977, 62, 40-46.

${ }^{43}$ T. Seideman and W. H. Miller, J. Chem. Phys., 1991, 95, 17681780.

44 S. Keshavamurthy and W. H. Miller, Chem. Phys. Lett., 1993, 205, 96-101.

45 R. Hernandez and W. H. Miller, Chem. Phys. Lett., 1993, 214, 129-136.

46 R. Hernandez, J. Chem. Phys., 1994, 101, 9534-9547.

${ }^{47}$ R. A. Marcus, Science, 1992, 256, 1523-1524.

48 D. J. Wales and R. S. Berry, J. Phys. B, 1991, 24, L351.

${ }^{49}$ R. J. Hinde, R. S. Berry and D. J. Wales, J. Chem. Phys., 1992, 96, 1376-1390.

${ }^{50}$ C. Amitrano and R. S. Berry, Phys. Rev. Lett., 1992, 68, 729-732.

${ }^{51}$ R. J. Hinde and R. S. Berry, J. Chem. Phys., 1993, 99, 29422963.

52 S. Kawai and T. Komatsuzaki, Phys. Chem. Chem. Phys., 2010, 12, 7626-7635.

53 S. Kawai and T. Komatsuzaki, Phys. Rev. Lett., 2010, 105, 048304.

${ }^{54}$ H. Mori, Prog. Theor. Phys., 1965, 33, 423-455.

55 K. Kawasaki, J. Phys. A, 1973, 6, 1289-1295.

${ }^{56}$ R. Zwanzig, Nonequilibrium Statistical Mechanics, Oxford University Press, London, 2001.

57 H. A. Kramers, Physica, 1940, 7, 284-304.

58 R. F. Grote and J. T. Hynes, J. Chem. Phys., 1980, 73, 27152732.

59 H. Sumi and R. A. Marcus, J. Chem. Phys., 1986, 84, 4894-4914.

${ }^{60}$ E. Pollak, H. Grabert and P. Hänggi, J. Chem. Phys., 1989, 91, 4073.

${ }^{61}$ A. M. Berezhkovskii, A. M. Frishman and E. Pollak, J. Chem. Phys., 1994, 101, 4778.

62 A. M. Berezhkovskii, V. Y. Zitserman, D. Y. Yang, J. Kuo and S. H. Lin, Physica A, 1998, 251, 399-429.

63 C. C. Martens, J. Chem. Phys., 2002, 116, 2516-2528.

64 T. Bartsch, R. Hernandez and T. Uzer, Phys. Rev. Lett., 2005, 95, 058301.

65 T. Bartsch, T. Uzer and R. Hernandez, J. Chem. Phys., 2005, 123, 204102.

66 T. Bartsch, T. Uzer, J. M. Moix and R. Hernandez, J. Chem. Phys., 2006, 124, 244310.

67 T. Bartsch, J. Chem. Phys., 2009, 131, 124121.

${ }^{68}$ R. Hernandez, T. Uzer and T. Bartsch, Chem. Phys., 2010, 370, 270-276.

${ }^{69}$ D. W. Oxtoby and S. A. Rice, J. Chem. Phys., 1976, 65, 16761683.

${ }^{70}$ M. J. Davis and S. K. Gray, J. Chem. Phys., 1986, 84, 5389-5411.

71 A. M. Berezhkovskii, E. Pollak and V. Y. Zitserman, J. Chem. Phys., 1992, 97, 2422-2437.

72 E. Pollak and P. Talkner, Phys. Rev. E, 1993, 47, 922-933.

73 E. Pollak, A. M. Berezhkovskii and Z. Schuss, J. Chem. Phys., 1994, 100, 334-339.

74 G. van der Zwan and J. T. Hynes, J. Chem. Phys., 1983, 78, 4174 
4185

75 E. Pollak, Chem. Phys. Lett., 1986, 127, 178-182.

76 S. Kawai and T. Komatsuzaki, J. Chem. Phys., 2009, 131, 224505.

77 S. Kawai and T. Komatsuzaki, J. Chem. Phys., 2009, 131, 224506.

78 S. Kawai and T. Komatsuzaki, Phys. Chem. Chem. Phys., 2010, 12, 7636-7647.

79 S. Kawai and T. Komatsuzaki, Phys. Chem. Chem. Phys., 2010, 12, 15382-15391.

${ }^{80}$ E. Ryter, J. Stat. Phys., 1987, 49, 751-765.

${ }^{81}$ M. M. Klosek, B. J. Matkowsky and Z. Schuss, Ber. Bunsenges. Phys. Chem., 1991, 95, 331-337.

${ }^{82}$ B. C. Dian, A. Longarte and T. S. Zwier, Science, 2002, 296, 2369-2373.

${ }^{83}$ K. Nakai, H. Kono, Y. Sato, N. Niitsu, R. Sahnoun, M. Tanaka and Y. Fujimura, Chemical Physics, 2007, 338, 127-134.

${ }^{84}$ K. Iwata, J. Raman Spectrosc., 2008, 39, 1512-1517.

${ }^{85}$ M. M. Millonas and C. Ray, Phys. Rev. Lett., 1995, 75, 11101113.

${ }^{86}$ S. Bhattacharya, S. K. Banik, S. Chattopadhyay and J. R. Chaudhuri, J. Math. Phys., 2008, 49, 063302.

87 J. R. Chaudhuri, G. Gangopadhyay and D. S. Ray, J. Chem. Phys., 1998, 109, 5565-5575.

${ }^{88}$ R. Hernandez and F. L. Somer, Jr., J. Phys. Chem. B, 1999, 103, 1064-1069.

89 R. Hernandez, J. Chem. Phys., 1999, 111, 7701-7704.

90 R. Hernandez and F. L. Somer, J. Phys. Chem. B, 1999, 103, 1070-1077.

${ }^{91}$ F. L. Somer and R. Hernandez, J. Phys. Chem. A, 1999, 103, 11004-11010.

92 F. L. Somer and R. Hernandez, J. Phys. Chem. B, 2000, 104, 3456-3462.

${ }_{93}$ M. Vogt and R. Hernandez, J. Chem. Phys., 2005, 123, 144109.

94 A. V. Popov and R. Hernandez, J. Chem. Phys., 2007, 126, 244506.
95 R. Zwanzig, J. Stat. Phys., 1973, 9, 215-220.

96 E. Cortés, B. J. West and K. Lindenberg, J. Chem. Phys., 1985, 82, 2708-2717.

${ }^{97}$ S. Kawai and T. Komatsuzaki, J. Chem. Phys., 2011, 134, 114523.

98 V. I. Mel'nikov and S. V. Meshkov, J. Chem. Phys., 1986, 85, 1018-1027.

99 J. S. Langer, Ann. Phys., 1969, 54, 258-275.

${ }^{100}$ K. Voigtlaender and H. Risken, Journal of Statistical Physics, 1985, 40, 397-429.

101 H.-X. Zhou, Chem. Phys. Lett., 1989, 164, 285-290.

102 E. Darve, J. Solomon and A. Kia, Proc. Nat. Acad. Sci., 2009, 106, 10884-10889.

${ }^{103}$ S. Kawai, A. D. Bandrauk, C. Jaffé, T. Bartsch, J. Palacián and T. Uzer, J. Chem. Phys., 2007, 126, 164306.

104 S. Kawai and T. Komatsuzaki, J. Chem. Phys., 2011, 134, 024317.

105 A. Y. T. Leung and Q. C. Zhang, J. Sound Vib., 2003, 266, 261279.

106 A. Deprit, Cel. Mech., 1969, 1, 12-30.

107 A. Lichtenberg and M. Lieberman, Regular and Chaotic Dynamics, Springer, 1992.

108 R. P. McRae, G. K. Schenter, B. C. Garrett, Z. Svetlicic and D. G. Truhlar, J. Chem. Phys., 2001, 115, 8460-8480.

109 I. S. Tolokh, G. W. N. White, S. Goldman and C. G. Gray, Mol. Phys., 2002, 100, 2351-2359.

110 M. Nagaoka, N. Yoshida and T. Yamabe, J. Chem. Phys., 1996, 105, 5431-5445.

111 J. J. Ruiz-Pernía, I. Tuñón, V. Moliner, J. T. Hynes and M. Roca, J. Am. Chem. Soc., 2008, 130, 7477-7488.

112 Single Molecule Biophysics: Experiments and Theories, $A d v$. Chem. Phys., ed. T. Komatsuzaki, M. Kawakami, S. Takahashi, H. Yang and R. J. Silbey, John-Wiley \& Sons, Inc., 2012, vol. 146. 